\title{
Nebular gas drag and co-orbital system dynamics
}

\author{
T. Chanut ${ }^{1}$, O. C. Winter $^{1}$, and M. Tsuchida ${ }^{2}$ \\ 1 São Paulo State University - UNESP, Grupo de Dinâmica Orbital \& Planetologia, CP 205, Guaratinguetá, \\ CEP 12516-410, SP, Brazil \\ e-mail: madamechanut@terra.com.br; ocwinter@feg.unesp.br \\ 2 São Paulo State University - UNESP, DCCE - IBILCE, São Jose do Rio Preto, SP, Brazil \\ e-mail: tsuchida@ibilce.unesp.br
}

Received 11 June 2007 / Accepted 7 December 2007

\section{ABSTRACT}

\begin{abstract}
Aims. We study trajectories of planetesimals whose orbits decay due to gas drag in a primordial solar nebula and are perturbed by the gravity of the secondary body on an eccentric orbit whose mass ratio takes values from $\mu_{2}=10^{-7}$ to $\mu_{2}=10^{-3}$ increasing ten times at each step. Each planetesimal ultimately suffers one of the three possible fates: (1) trapping in a mean motion resonance with the secondary body; (2) collision with the secondary body and consequent increase of its mass; or (3) diffusion after crossing the orbit of the secondary body.

Methods. We take the Burlirsh-Stoer numerical algorithm in order to integrate the Newtonian equations of the planar, elliptical restricted three-body problem with the secondary body and the planetesimal orbiting the primary. It is assumed that there is no interaction among planetesimals, and also that the gas does not affect the orbit of the secondary body.

Results. The results show that the optimal value of the gas drag constant $k$ for the $1: 1$ resonance is between 0.9 and 1.25 , representing a meter size planetesimal for each AU of orbital radius. In this study, the conditions of the gas drag are such that in theory, L4 no longer exists in the circular case for a critical value of $k$ that defines a limit size of the planetesimal, but for a secondary body with an eccentricity larger than 0.05 when $\mu_{2}=10^{-6}$, it reappears. The decrease of the cutoff collision radius increase the difusions but does not affect the distribution of trapping. The contribution to the mass accretion of the secondary body is over $40 \%$ with a collision radius $0.05 R_{\text {Hill }}$ and less than $15 \%$ with $0.005 R_{\text {Hill }}$ for $\mu_{2}=10^{-7}$. The trappings no longer occur when the drag constant $k$ reachs 30 . That means that the size limit of planetesimal trapping is $0.2 \mathrm{~m}$ per AU of orbital radius. In most cases, this accretion occurs for a weak gas drag and small secondary eccentricity. The diffusions represent most of the simulations showing that gas drag is an efficient process in scattering planetesimals and that the trapping of planetesimals in the 1:1 resonance is a less probable fate. These results depend on the specific drag force chosen.
\end{abstract}

Key words. planets and satellites: formation - minor planets, asteroids - celestial mechanics

\section{Introduction}

In 1788, in Analytical Mechanics, Lagrange demonstrated that in the restricted three-body problem, distinct particular solutions exist. These particular solutions are known as Lagrangian points and in the bi-dimensional system we have five Lagrangian points. Three of these are the collinear points (L1, L2 and L3) that are the points located on the line that joins the primary and secondary body, and the other two (L4 and L5) are the triangular points, since they are on the vertices of the equilateral triangles formed jointly with the two bodies. From the theoretical point of view it is known that tadpole orbits could exist from oscillations around L4 or L5, while horseshoe orbits could exist from oscillations around L4, L3 and L5 (e.g. Brown $\&$ Shook 1964). Confirming the theoretical demonstration of the existence of these types of steady orbits, in 1906 was observed the asteroid 588 Achille, librating around L4. Today hundreds of asteroids, called Trojans, have been discovered librating around L4 and L5. In 1980, images from the Voyager spacecraft showed that in the Saturn-Tethys system, two satellites librate around the Lagrangian points. One of them, called Telesto, oscillates around L4. The other, Calypso, oscillates around L5. Also in 1980, Voyager found a singular pair of co-orbital satellites in the Saturn system: Janus and Epimetheus (the Janus mass is approximately four times the mass of Epimetheus) and in the Saturn-Dione system found a satellite of mass much less than Dione, called Helene, librating around L4. More recently, Cassini found Polideuces in the Saturn-Dione system librating around L5. There are various ideas concerning the origin of the co-orbital systems. Yoder (1979) suggested three possibilities: first, that Jupiter captured the asteroids throughout the whole history of its growth. Second, these objects originally may have been satellites of Jupiter and through some process (collision or escape) moved through the interior Lagrangian point. Finally, this population of co-orbital objects may have been trapped in a mean motion resonance with the secondary body. The stability of Trojan asteroid orbits when submitted to the dissipative forces such as drag due to the gas of the primitive solar nebula has been a focus of divergences of point of view found in literature (Peale 1993). Murray (1994) analyzed the stability for small librations showing that a certain class of drag forces lead to asymptotical stability. In particular, Murray demonstrated that this occurs for a force proportional to the velocity in the inertial system. Kary \& Lissauer (1995) reviewed the third idea of Yoder and they suggested that in the case of moderate or high eccentricities of the secondary body, the capture is feasible. More recently, Morbidelli et al. (2005) suggested that the Trojans could have formed in more distant regions and been subsequently captured into co-orbital motion with Jupiter during the time when the giant planets migrated. 
The purpose of this work is to investigate how the $1: 1$ resonance captures occur. We analyse the evolution of the semimajor axis and eccentricity of the planetesimal, and what conditions are favorable for this type of capture. For example, the angular distribution between the planetesimal and the secondary body at the moment of its entrance in the co-orbital region, the average of the longitude between the planetesimal and the secondary body during the trapping in the 1:1 resonance, the values of orbital eccentricity of the secondary body, as well as the values of the drag constant (see Sect. 2.2 for a definition of $k$ ). The mass ratio $\mu_{2}=10^{-6}$ for the secondary body is the aim of the work but we set the values of the drag constant for $\mu_{2}=10^{-7}$ to $\mu_{2}=10^{-3}$, according to Murray's critical value $k=0.7265\left(v_{\mathrm{K}}^{2} / v_{\text {gas }}^{2}\right) \mu_{2}$ (Murray 1994) where L4 no longer exists in the circular case. On the other hand we establish statistics of the collision rate for two cutoff collision radii for a close approach of the planetesimal of less than $0.005 R_{\text {Hill }}$ and $0.05 R_{\text {Hill }}$ from the secondary body, which implies in an increase of the mass of the secondary body. We also study the statistics of the diffusion rate that represents the planetesimals crossing the coorbital region towards the primary body, or being scattered by the secondary body. In Sect. 2 we describe the equations of motion and the nebular model that we use in this paper. We then present how the simulations are set in Sect. 3 and discuss the simulation results in Sect. 4. Conclusions are given in Sect. 5.

\section{Dynamical system}

In reality, each small planetesimal is subject to a number of external disturbances of different magnitudes that are added to the gravitational forces. Some studies have been performed considering the effect of radiation pressure as a leading perturbation (Colombo et al. 1966; Schuerman 1980; Simmons et al. 1985) and others the dynamic effect of nebular drag forces (Peale 1993; Murray 1994; Kary \& Lissauer 1995). Here, we consider the dynamic effect of the nebular drag forces on a planetesimal in the elliptic restricted three-body problem.

\subsection{Equations of motion}

Let us consider the motion of a small planetesimal of negligible mass moving under the gravitational influence of two bodies with masses $m_{1}$ and $m_{2}\left(m_{1}>m_{2}\right)$. We assume that these bodies have elliptic orbits about their common center of mass $O$, and the planetesimal cannot affect these orbits. Let $\mu_{1}=m_{1} /\left(m_{1}+m_{2}\right)$ and $\mu_{2}=m_{2} /\left(m_{1}+m_{2}\right)$ and consider the inertial coordinate system $(\xi, \eta)$. Let the coordinates of the two masses in this reference frame be $\left(\xi_{1}, \eta_{1}\right)$ and $\left(\xi_{2}, \eta_{2}\right)$. Adopting the distance between the two masses (semimajor axis) as unity and total mass unity, the mean motion of the two-body system becomes unity, and the equations of the motion of the planetesimal are

$$
\begin{gathered}
\ddot{\xi}=\mu_{1} \frac{\xi_{1}-\xi}{r_{1}^{3}}+\mu_{2} \frac{\xi_{2}-\xi}{r_{2}^{3}}+F_{\xi}(\xi, \eta, \dot{\xi}, \dot{\eta}), \\
\ddot{\eta}=\mu_{1} \frac{\eta_{1}-\eta}{r_{1}^{3}}+\mu_{2} \frac{\eta_{2}-\eta}{r_{2}^{3}}+F_{\xi}(\xi, \eta, \dot{\xi}, \dot{\eta}),
\end{gathered}
$$

where

$r_{1}^{2}=\left(\xi_{1}-\xi\right)^{2}+\left(\eta_{1}-\eta\right)^{2}$

$r_{2}^{2}=\left(\xi_{2}-\xi\right)^{2}+\left(\eta_{2}-\eta\right)^{2}$

and $F_{\xi}$ and $F_{\eta}$ are the $\xi$ and $\eta$ components of the gas drag which can be expressed as a function of the position and velocity in the inertial frame.

\subsection{Nebular drag}

As the nebular gas turns around the primary body with an angular velocity smaller than the Keplerian value, a planetesimal orbiting with a Keplerian velocity will suffer a headwind that continuously reduces its orbital energy and angular momentum (Weidenchilling 1977). The resistance to the motion of a body due to the gas is produced by differences of pressure and friction. When the relative velocity $v_{\text {rel }}$ is high, the pressure differences dominate, thus the resistance is proportional to the dynamic pressure $\left(\rho v_{\text {rel }}^{2} / 2\right)$ or the drag force $F_{\mathrm{d}} \propto \rho v_{\text {rel }}^{2} S$, where $S$ is the cross section. At low velocity the friction dominates, then the resistance is proportional to $v_{\text {rel }}$. Generally, we adopt the $v_{\text {rel }}^{2}$ representation and use a variable drag coefficient to accommodate all relative velocities. Thus, we can write for a spherical planetesimal (e.g. Prantl 1952)

$$
\frac{\boldsymbol{F}_{\mathrm{d}}}{m_{\mathrm{p}}}=-\frac{c_{\mathrm{d}} \pi R^{2} \rho}{2 m_{\mathrm{p}}} v_{\text {rel }} \boldsymbol{V}_{\text {rel }}=-\frac{3 c_{\mathrm{d}} \rho}{8 \rho_{\mathrm{p}} R} v_{\text {rel }} \boldsymbol{V}_{\text {rel }}
$$

where $m_{\mathrm{p}}$ is the planetesimal mass, $R$ the planetesimal radius, $\rho_{\mathrm{P}}$ is the planetesimal density, $\rho$ the gas density and $c_{\mathrm{d}}$ the drag coefficient which is a function of the Reynolds number. Malhotra (1993) used a simplified form of Pealet's drag force where $c_{\mathrm{d}}=$ 0.5 is constant. It is customary to adopt $\rho=10^{-10} \mathrm{~g} / \mathrm{cm}^{3}$ and $\rho_{\mathrm{P}}=2 \mathrm{~g} / \mathrm{cm}^{3}$ (Malhotra 1993; Kary \& Lissauer 1995; Marzari $\&$ Scholl 1997). In these units, the value of the drag constant $k=(3 / 8) c_{\mathrm{d}}\left(\rho / \rho_{\mathrm{P}}\right) / R_{\mathrm{P}}$ is 0.014 per $\mathrm{AU}$ of orbital radius for $R_{\mathrm{P}}=$ $100 \mathrm{~m}$. Finally,

$\boldsymbol{V}_{\text {rel }}=\boldsymbol{V}-\boldsymbol{V}_{\mathrm{gas}}=(\dot{\xi}+\eta \Omega) \boldsymbol{e}_{\xi}+(\dot{\eta}-\xi \Omega) \boldsymbol{e}_{\eta}$

where $V$ is the planetesimal velocity with components $\dot{\xi}$ and $\dot{\eta}$ along the orthogonal directions denoted by the unit vectors $\boldsymbol{e}_{\xi}$ and $\boldsymbol{e}_{\eta}$ on the midplane of the nebula and $\Omega$ is the gas angular velocity. All the motions are confined to the midplane of the nebula.

\section{Numerical simulations}

The Burlirsh-Stoer numerical algorithm integrates the Newtonian equations in the planar, elliptical restricted threebody problem with the secondary body and the planetesimal orbiting the primary. The described model of nebular drag was presented by Peale (1993). It is assumed that there is no interaction among planetesimals, and also that the gas does not affect the orbit of the secondary body. The initial position of the secondary body is taken always at its pericenter and the planetesimals are disposed in a ring with width 10 to 30 Hill spheres $\left(R_{\text {Hill }}=\left(\mu_{2} / 3\right)^{1 / 3}\right.$ since $a=1$, where $a$ is the secondary body semimajor axis). We chose $\mu_{2}=10^{-7}$ up to $\mu_{2}=10^{-3}$ because it represents a large number of relative mass systems. The initial positions of planetesimals are at each $30^{\circ}$ in the ring and initial velocities are given by the Keplerian velocity. The simulation time is about $10^{5}$ units of time, or about 16000 secondary body orbital periods, and it is enough for the planetesimals to define their final destiny.

As a planetesimal migrates toward the secondary body it may have one of several possibilities shown in Fig. 1. It can be trapped in an exterior resonance having or not a close encounter with the secondary body. It can also cross the secondary body orbit and continue its migration toward the primary body. Alternatively, the encounter could result in the capture of the planetesimal into a stable libration within the 1:1 resonance. The 
Table 1. Distribution of trapping in the 1:1 resonance, collisions, diffusions and other events related to the dynamics of a planetesimal submitted to the effect of gaseous drag. The distribution is for several mass ratios and for the cutoff collision radius $0.005 R_{\text {Hill }}$ and $0.05 R_{\text {Hill }}$.

\begin{tabular}{cccccc}
\hline \hline Mass ratio & Collision radius & Co-orbitals & Collisions & Diffusions & Others \\
\hline$\mu_{2}=10^{-7}$ & $0.005 R_{\text {Hill }}$ & $3 \%$ & $12 \%$ & $68 \%$ & $17 \%$ \\
& $0.05 R_{\text {Hill }}$ & $3 \%$ & $43 \%$ & $41 \%$ & $13 \%$ \\
$\mu_{2}=10^{-6}$ & $0.005 R_{\text {Hill }}$ & $8 \%$ & $7 \%$ & $79 \%$ & $6 \%$ \\
& $0.05 R_{\text {Hill }}$ & $7 \%$ & $28 \%$ & $59 \%$ & $6 \%$ \\
$\mu_{2}=10^{-5}$ & $0.005 R_{\text {Hill }}$ & $5 \%$ & $4 \%$ & $88 \%$ & $3 \%$ \\
& $0.05 R_{\text {Hill }}$ & $5 \%$ & $23 \%$ & $69 \%$ & $3 \%$ \\
$\mu_{2}=10^{-4}$ & $0.005 R_{\text {Hill }}$ & $<1 \%$ & $1 \%$ & $98 \%$ & $<1 \%$ \\
& $0.05 R_{\text {Hill }}$ & $<1 \%$ & $24 \%$ & $75 \%$ & $<1 \%$ \\
$\mu_{2}=10^{-3}$ & $0.005 R_{\text {Hill }}$ & - & $23 \%$ & $77 \%$ & - \\
& $0.05 R_{\text {Hill }}$ & - & $27 \%$ & $73 \%$ & - \\
\hline
\end{tabular}

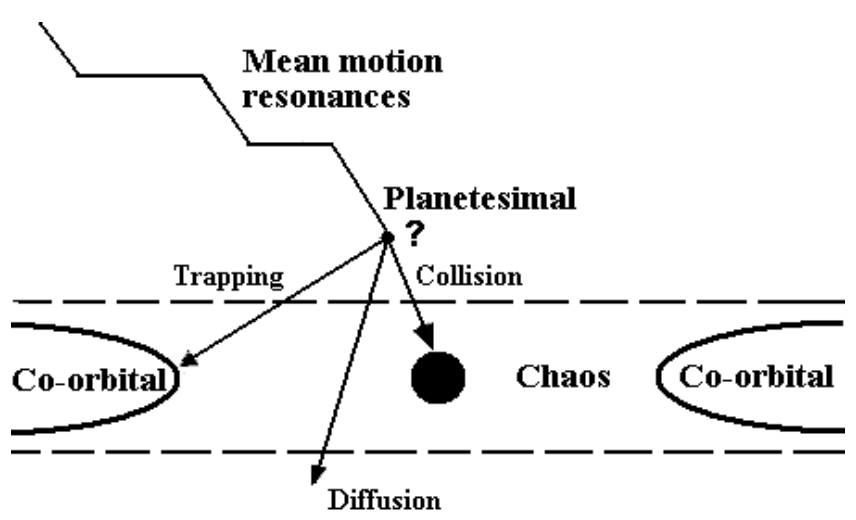

Fig. 1. Illustration showing the several possibilities of trajectory evolution of a planetesimal when it comes close to the secondary body co-orbital zone.

planetesimal could also collide with the secondary body, increasing its mass.

If the planetesimal remains orbiting the primary body with a semimajor axis within $1 R_{\text {Hill }}$ around the secondary body's orbit then it is assumed to be trapped in the $1: 1$ resonance. We choose two cutoff collision radii $\left(<0.005 R_{\text {Hill }}\right)$ and $\left(<0.05 R_{\text {Hill }}\right)$. If the planetesimal comes too close to the secondary body center (collision radius) then the integration is stopped, and a collision is assumed. We consider the diffusion if the planetesimal semimajor axis $a_{\mathrm{P}}$ becomes smaller than 0.9 which is away from $1 R_{\text {Hill }}$ below the co-orbital semimajor axis when $\mu_{2}=10^{-3}$. In our study, theoretically (Murray 1994) for the case of a secondary body with a circular orbit, L4 and L3 are displaced and disappear when $k>0.7265\left(v_{\mathrm{K}}^{2} / v_{\text {gas }}^{2}\right) \mu_{2}$. For example, if $v_{\text {gas }} / v_{\mathrm{K}}=0.005$ and $\mu_{2}=10^{-6}$, all the values of the gas drag constant larger than $k \approx 0.029$ satisfy this condition. This means that L4 no longer traps planetesimals smaller than $0.05 \mathrm{~km}$ per AU. At $5 \mathrm{AU}$ from the Sun, the critical value of $R_{\mathrm{P}}$ in this case is $250 \mathrm{~m}$. When $\mu_{2}=10^{-7}$, the critical value of $k$ is 0.0029 and for $\mu_{2}=10^{-3}, k$ is 29. Thus we can take for the initial condition of $k$ from 0.0025 to 0.1 with intervals of 0.0025 for $\mu_{2}=10^{-7}, 0.025$ to 1.0 with intervals of 0.025 for $\mu_{2}=10^{-6}, 0.25$ to 10 with intervals of 0.25 for $\mu_{2}=10^{-5}$, etc. For the eccentricity of the secondary body, we take values from 0 to 0.7 with intervals of 0.07 .

\section{Results}

In Table 1, we present our numerical data on the trapping in the 1:1 resonance, collisions, diffusions and other events related to the dynamics of a planetesimal in a gaseous nebula. The first column gives the mass ratio of the secondary body. We list the percentage of events for two different cutoff collision radii, $0.005 R_{\text {Hill }}$ and $0.05 R_{\text {Hill }}$. The co-orbitals column represents the percentage of bodies trapped in the 1:1 resonance. The results show that the mass ratio where the largest number of trapping occurs $(8 \%)$ for $\mu_{2}=10^{-6}$ and decreases to less than $1 \%$ for $\mu_{2}=10^{-4}$. When $\mu_{2}=10^{-3}$, trapping in the $1: 1$ resonance does not occur. The collisions column shows that the choice of the cutoff collision radius has an importance in relation to the accretion rate while it seems to be unimportant for the trapping results. Furthermore the collision rate is higher when the secondary body mass is lower. For a mass ratio of $\mu_{2}=10^{-7}$ it represents $44 \%$ for $0.05 R_{\text {Hill }}$ and $12 \%$ for $0.005 R_{\text {Hill }}$. These numbers decrease below $25 \%$ and $1 \%$ respectively when $\mu_{2}=10^{-4} \cdot \mu_{2}=10^{-3}$ is a special case because the cutoff collision radius does not change the results and a particular approach will be necessary. The diffusions represent the larger part of the simulations and increase when the cutoff collision radius decreases. We note other events: the resonances external to the co-orbital region or the fact that the planetesimal did not pass the limit to be considered diffusion by the end of the simulation.

In Tables 2 and 3, we list our numerical data relative to the values of the drag constant $k$ for each mass ratio. The second column represents the extreme values of the intervals chosen relative to Murray's critical limit. The cutoff collision radius in Table 2 is $0.005 R_{\text {Hill }}$ and in Table $3,0.05 R_{\text {Hill }}$. If we consider only the gas drag constant $k$ for all the values of $e$ as shown in Tables 2 and 3, we can affirm that the optimal value of $k$ for the $1: 1$ resonance trapping is between 0.9 and 1.25 , representing a meter size planetesimal per AU. This value represents Murray's critical limit when $\mu_{2}$ is larger than $10^{-6}$ and smaller than $10^{-5}$. For $\mu_{2}=10^{-7}$, we can suppose that part of the material trapped in an external resonance may collide with the secondary body, increasing its rate when $k<0.0125$. So, we can affirm that the accretion rate is higher when the gas drag constant is near the limit defined by Murray for each mass ratio. This impact probability generally decreases with increasing drag rate $k$, but this dependency is smaller when the cutoff collision radius is larger, as shown in Table 3. This will be explained later. In the opposite case, the diffusions are higher when the cutoff collision radius is $0.005 R_{\text {Hill }}$. This is more than $90 \%$ when $k>9.0$ and the dependency of $k$ is greater for a lower cutoff collision radius.The external resonances and other events do not depend on the cutoff collision radius. At each mass ratio these events are insignificant when the drag rate is higher. This means that the majority of planetesimals of large dimensions have collided or been trapped in some external resonance while the planetesimals of small dimensions have crossed the co-orbital zone. 
Table 2. Distribution of trapping in the 1:1 resonance, collisions, diffusions and other events related to the dynamics of a planetesimal submitted to the effect of gaseous drag. The distribution is for the extreme values of the drag constant $k$ relative to several mass ratios and for the cutoff collision radius $0.005 R_{\text {Hill }}$.

\begin{tabular}{cccccc}
\hline \hline Mass ratio & Drag constant & Co-orbitals & Collisions & Diffusions & Others \\
\hline$\mu_{2}=10^{-7}$ & $k<0.0125$ & $1 \%$ & $9 \%$ & $7 \%$ & $83 \%$ \\
& $k>0.09$ & $3 \%$ & $7 \%$ & $89 \%$ & $1 \%$ \\
$\mu_{2}=10^{-6}$ & $k<0.125$ & $4 \%$ & $8 \%$ & $35 \%$ & $53 \%$ \\
& $k>0.9$ & $6 \%$ & $4 \%$ & $90 \%$ & - \\
$\mu_{2}=10^{-5}$ & $k<1.25$ & $6 \%$ & $8 \%$ & $58 \%$ & $28 \%$ \\
& $k>9.0$ & $2 \%$ & $2 \%$ & $96 \%$ & - \\
$\mu_{2}=10^{-4}$ & $k<12.5$ & $1 \%$ & $2 \%$ & $94 \%$ & $3 \%$ \\
& $k>90.0$ & - & $1 \%$ & $99 \%$ & - \\
\hline
\end{tabular}

Table 3. Distribution of trapping in the 1:1 resonance, collisions, diffusions and other events related to the dynamics of a planetesimal submitted to the effect of gaseous drag. The distribution is for the extreme values of the drag constant $k$ relative to several mass ratios and for the cutoff collision radius $0.05 R_{\text {Hill }}$.

\begin{tabular}{cccccc}
\hline \hline Mass ratio & Drag constant & Co-orbitals & Collisions & Diffusions & Others \\
\hline$\mu_{2}=10^{-7}$ & $k<0.0125$ & $<1 \%$ & $30 \%$ & $<1 \%$ & $69 \%$ \\
& $k>0.09$ & $2 \%$ & $34 \%$ & $63 \%$ & $1 \%$ \\
$\mu_{2}=10^{-6}$ & $k<0.125$ & $4 \%$ & $26 \%$ & $19 \%$ & $51 \%$ \\
& $k>0.9$ & $5 \%$ & $18 \%$ & $77 \%$ & - \\
$\mu_{2}=10^{-5}$ & $k<1.25$ & $6 \%$ & $30 \%$ & $37 \%$ & $28 \%$ \\
& $k>9.0$ & $2 \%$ & $17 \%$ & $81 \%$ & - \\
$\mu_{2}=10^{-4}$ & $k<12.5$ & $1 \%$ & $53 \%$ & $43 \%$ & $3 \%$ \\
& $k>90.0$ & - & $10 \%$ & $90 \%$ & - \\
\hline
\end{tabular}

The results show that the greater number of trappings in the $1: 1$ resonance occur when $\mu_{2}=10^{-6}$. The drag conditions, in this case, seem to contribute more to the trapping. For this reason we have taken as the main goal of our work this mass ratio for the secondary body. Peale (1993) shows that the symmetry between L4 and L5 is broken in the presence of gaseous drag with the secondary body in a noncircular orbit. As did Kary \& Lissauer (1995), we choose values of the constant of drag that are high enough so that L4 and L3 disappear in the circular case (Murray 1994). However, one of our goals is to show what happens with the planetesimal at the moment of trapping in the $1: 1$ resonance, when, decaying in its orbit, it comes near to the secondary body. In Fig. 2, two examples are shown comparing the time variations of semimajor axis, eccentricity and longitude when the mass ratio of the secondary body is $\mu_{2}=10^{-6}$. The capture in an external resonance increase the eccentricity and the probability of the planetesimal to encounter the secondary body. When the planetesimal crosses the secondary body's orbit, there is a large increase in its eccentricity, which can be explained by an exchange of angular momentum with the secondary body. After that, the oscillations of its eccentricity and semimajor axis diminish, stabilizing its orbit. Figure 2 shows that, if the secondary body's eccentricity is 0.07 , the L4 point reappears. Here, $\mu_{2}=10^{-6}$. We can see that the number of oscillations during and after the trapping around L4 is smaller than around L5. This can be explained by the fact that the friction due to the drag is smaller $(k=0.025)$, characterizing a larger size for the planetesimal (Marzari \& Sholl 1997). Now, the goal is to know what the angles are between the planetesimal and the secondary body that favor the trapping in the $1: 1$ resonance after the encounter. A first analysis shows that, in the majority of the trappings, the entrance of the planetesimal in the co-orbital region occurs close to the secondary body in more than $70 \%$ of the cases (Fig. 3). These entrances occur in the region between $0^{\circ}$ and $30^{\circ}$. Increasing the eccentricity, Fig. $3 \mathrm{~d}$ shows that the attracting region around the L5 point increases when L5 starts to move in the direction of the secondary body. When the eccentricity reaches its maximum (0.07), the region of the largest number of planetesimals is situated on the other side of the secondary body, between $-30^{\circ}$ and $0^{\circ}$. It also seems that the feeding region is larger to the side of the L5 point when the eccentricity of the secondary body is larger. This corroborates Kary \& Lissauer (1995) affirmation that the secondary body's epicyclical motion due to the eccentricity increases the relative velocity between the planetesimal and the secondary body and enlarges the size of the feeding region. Increasing the size of the feeding region gives the planetesimal more opportunities to have close encounters with the secondary body. The average of the longitude between the planetesimal and the secondary body during the trapping shows if the planetesimal was captured by the point L5 or L4. From Figs. 4a, b, we see that the majority of captures is around the L5 point. From Figs. 4c, d, we note, when the eccentricity is greater than 0.05 , that some averages of the longitude are between $60^{\circ}$ and $90^{\circ}$, characterizing a trapping around the L4 point and $120^{\circ}$ characterizing a horseshoe orbit. These orbits appear in an angular position that is lesser than the critical value established by Murray (1994), $108^{\circ}$ for $k$ larger than aproximatly 0.025 when $\mu_{2}=10^{-6}$. Namouni \& Murray (2000) have shown that the eccentricity displaces the L4 point towards the secondary body and the L5 point moves in the opposite direction. The greater the eccentricity the greater the displacement of the Lagrangian points in the direction opposite to the displacement due to the gas drag. Thus, it is evident that from an eccentricity of 0.05 this displacement become sufficiently large for the re-appearance of trapping around L4.

Kary \& Lissauer (1995) studied in an analytical way the probability of impact for a planetesimal approaching a secondary body in two idealized limits: (1) the gravity force of the secondary body can be neglected (1-body approximation) and (2) the gravity force of the secondary body only affects the planetesimal when the two are close enough that the effect of the primary body on the planetesimal's motion relative to the secondary body 

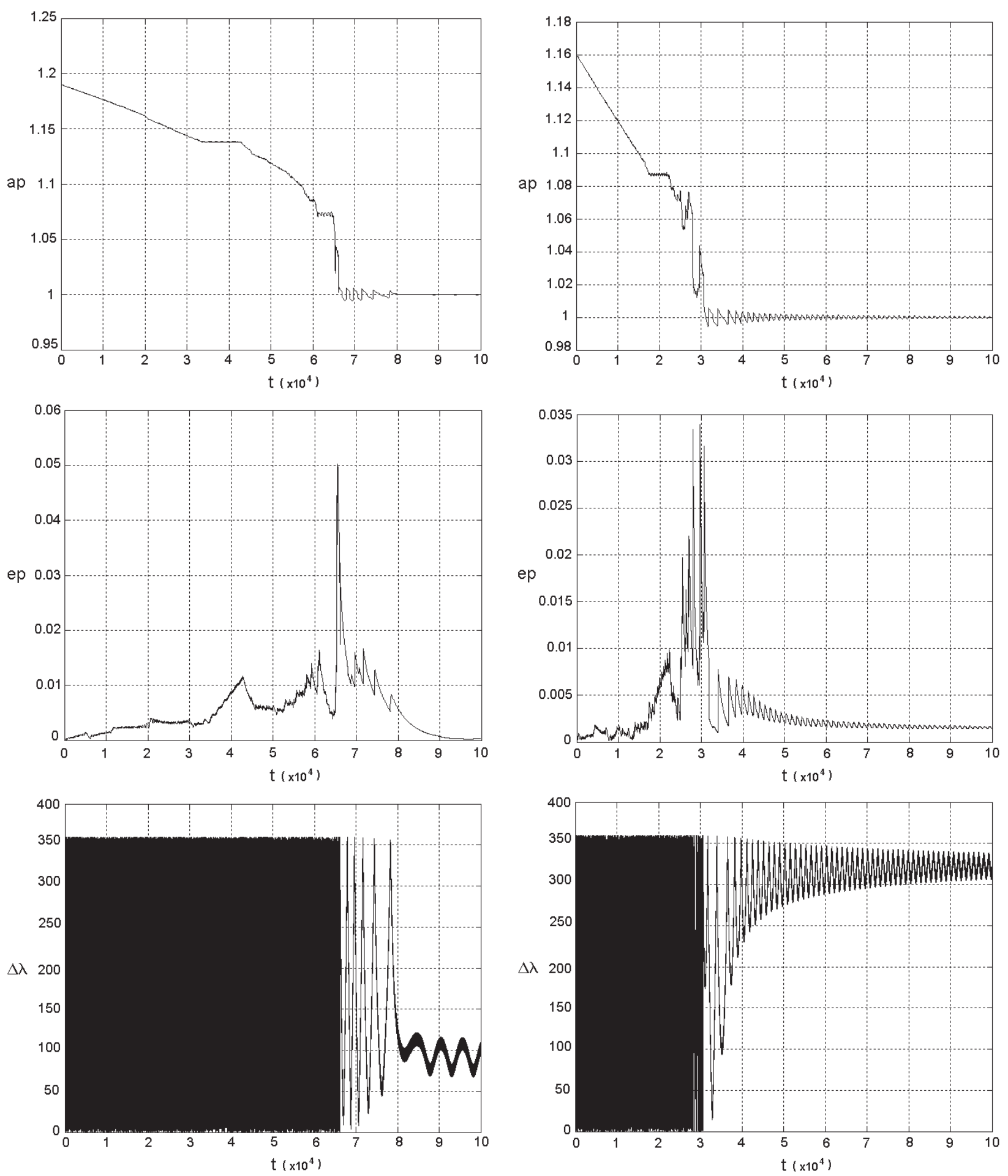

Fig. 2. Temporal evolution of the semi-major axis (top), the eccentricity (middle) and the longitude (bottom) of a planetesimal. In this case $\mu_{2}=10^{-6}$ for the secondary body and the initial conditions are $r_{0}=1.16, e_{2}=0.056$ and $k=0.075$ (left column), $r_{0}=1.19, e_{2}=0.07$ and $k=0.025$ (right column).

can be neglected (2+2-body approximation). Figure 5 illustrates well this behavior when the approaches to the secondary body increase its eccentricity in a significant way, reducing the impact probability per conjunction. In the case of a secondary body in an eccentric orbit, we have a relative velocity high enough to increase the disturbance effect of the secondary body on the planetesimal's eccentricity, allowing a 2+2-body approximation. There, the planetesimal no longer has an instantaneous circular orbit, as is shown in Fig. 5. But as the drag reduces the eccentricity of the planetesimal between the conjunctions, the greater the drag, the more quickly the eccentricity will be reduced. This means that the drag decreases the disturbance effect of the secondary body, needing the full three-body treatment. In reality, the 3-body effect comes to dominate the accretion behavior and numerical models become important. The distribution of trappings, collisions and diffusions relative to the secondary body eccentricity (Fig. 6) and the gas drag (Fig. 8) for the two cutoff collision radii when the mass ratio is $10^{-6}$ shows that the 

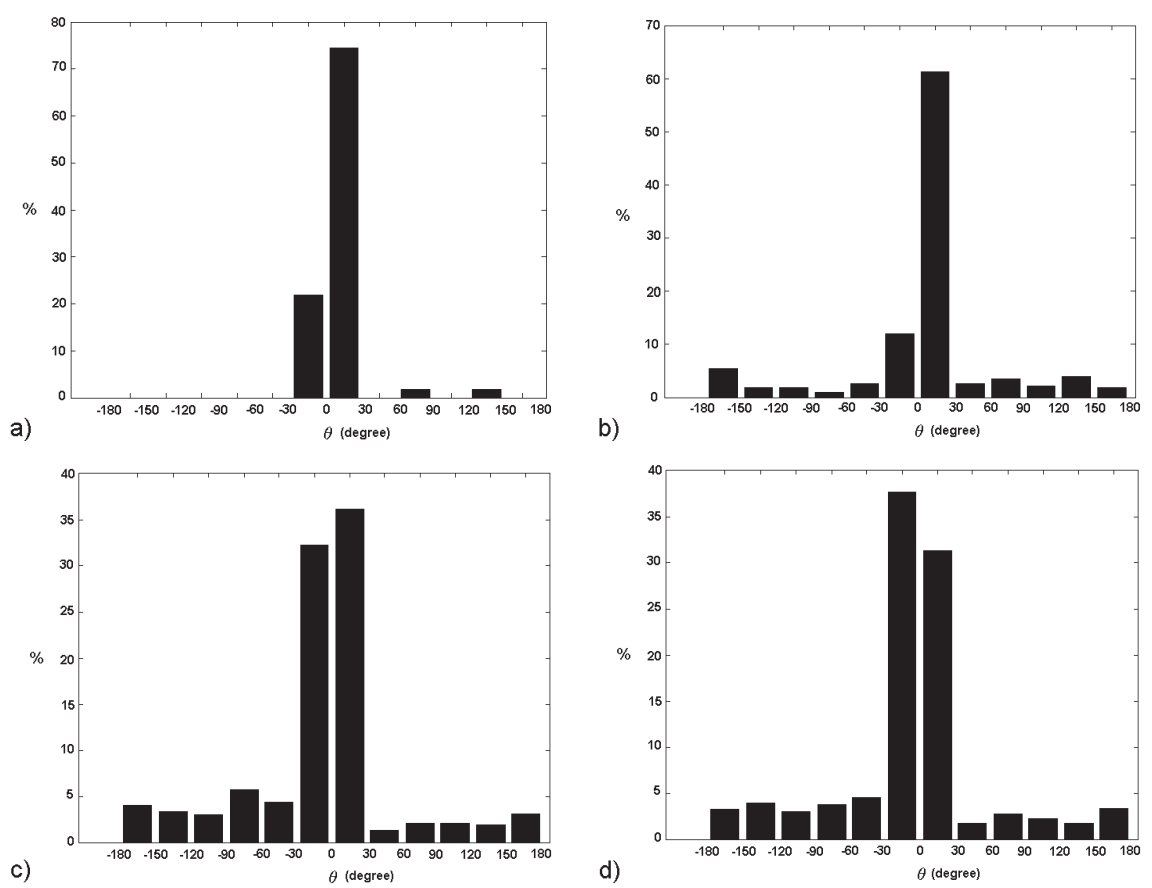

Fig. 3. Distribution of the angle $\theta$ between the planetesimal and the secondary body at the moment of its entrance in the co-orbital region, when it was trapped in the 1:1 resonance. They are for all the values of $0.025<k<$ 1.0 and for the eccentricities of the following secondary body's orbits when $\mu_{2}=10^{-6}$ : a) $e=0.007$; b) $e=0.014$ c) $e=0.063$ and d) $e=0.07$.

Fig. 4. Average of the longitude $\langle\Delta \lambda\rangle$ between the planetesimal and the secondary body during the trapping in the $1: 1$ resonance, for all the values of $0.025<k<1.0$ and for the eccentricities of the following secondary body's orbits when $\mu_{2}=10^{-6}$ : a) $e=0.007$; b) $e=0.014$; c) $e=0.063$ and d) $e=$ 0.07 .

d)
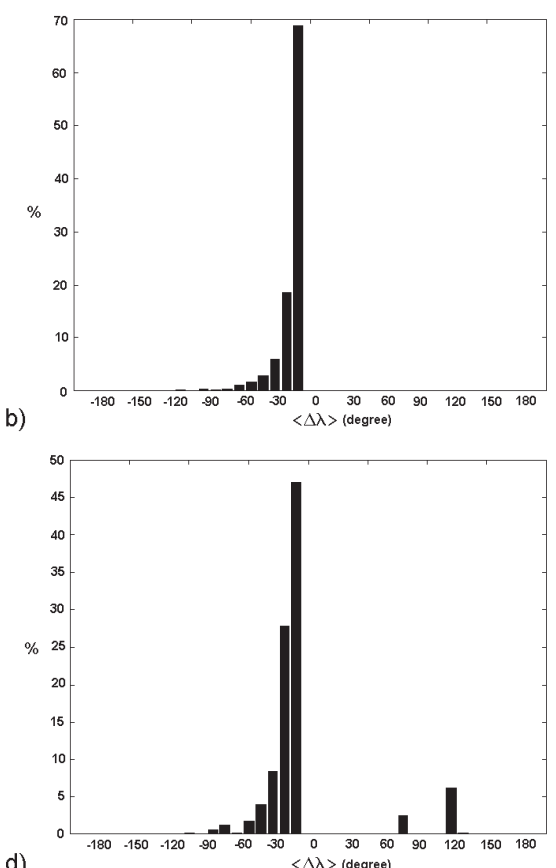

c)

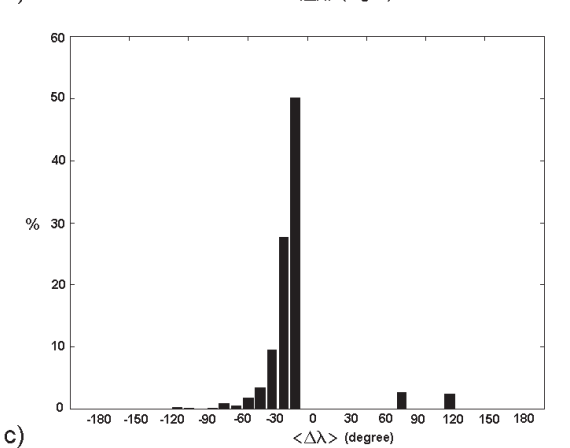

cutoff collision radius for these two values do not change the behavior of the distribution. Now, observing Fig. 6, we notice that the number of trappings is maximum for $e=0.021$ and $k=0.3$, decreasing almost linearly when their values increase. In our model, these conditions seem to favor the co-orbital trappings. We also notice that as the value of the eccentricity increases the distribution of the three events tends to be equal, but the co-orbital events dominate for $e>0.021$ (Fig. 6). The trapping distributions for $10^{-5}$ and $10^{-4}$ (Fig. 7) occurs for a higher eccentricity of the secondary body. For example, when $\mu_{2}=10^{-4}$ the trappings begin over 0.04 . The maximum trapping occurs when $e=0.05$ for $\mu_{2}=10^{-5}$ and is larger than 0.07 for $\mu_{2}=10^{-4}$. Looking at the collision rate we see that it is higher ( $40 \%$ of the cases) when $e=0$ to $e=0.014$, which means the eccentricity increases the relative velocity between the secondary body and the planetesimal and decreases the accretion rate per encounter (Fig. 6). At a very low value of $k$ the eccentricity determines the distribution but when $k$ is higher the dependency of the eccentricity does not occur (Fig. 7b). In Figs. 8 and 9 we notice that the gas drag diminishes the trapping distribution. The higher the drag rate, more quickly the decrease of the trappings occurs. Figure 9 shows that the trappings do not occur when $k$ reaches the limit of 30 . That means that the size limit of the planetesimal trapping is $0.2 \mathrm{~m}$ per AU. For example, at $5 \mathrm{AU}$ from the Sun, Jupiter might not capture planetesimals smaller than $1 \mathrm{~m}$. If we compare the collision distribution relative to the gas drag constant $k$, independent of the eccentricity of the secondary body, we see a maximum ( $15 \%$ of cases) when $k=0.2$ and a linear decrease from this value (Fig. 8). The diffusion distribution is different to the collisions. This distribution increases with the increase of the value of $k$ as is shown in Figs. 8 and 9. The diffusion rate depends on the value of the gas drag constant 

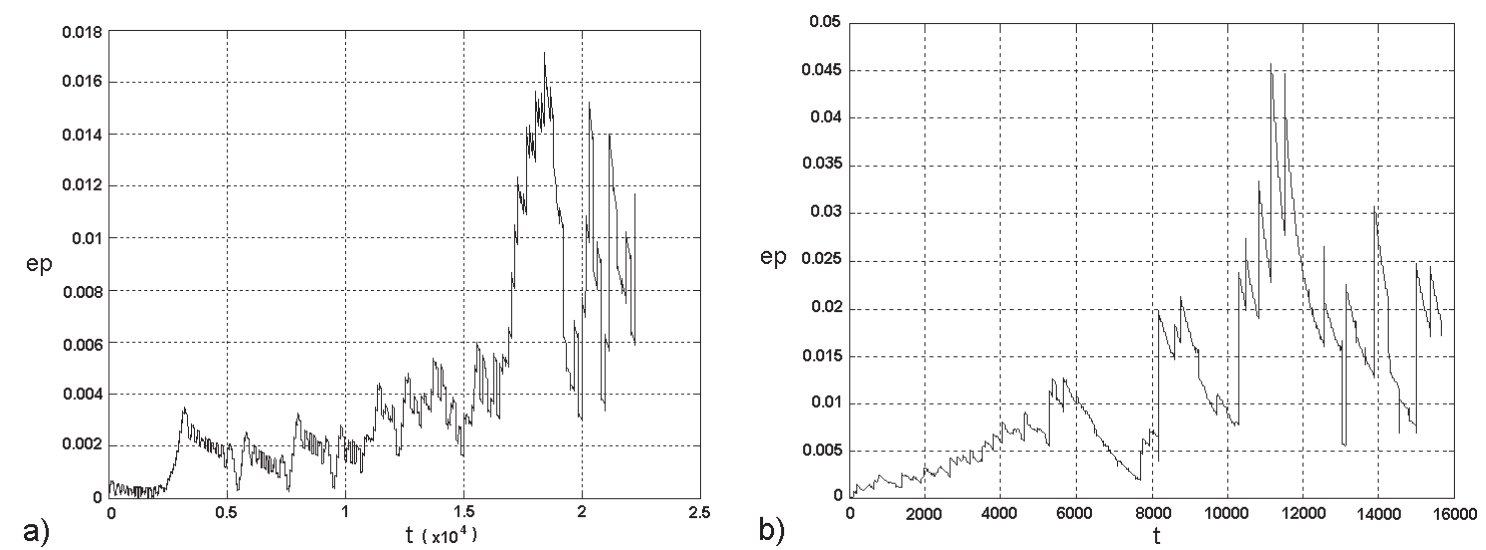

Fig. 5. Behavior of the eccentricity of the planetesimal during its process of approach before the collision with the secondary body. The initial conditions in this case are $r_{0}=1.08$ for the planetesimal, and $k=0.050$ for the constant of the gas drag and $\mu_{2}=10^{-6}$. The eccentricity of the secondary body: a) $e=0$ and b) $e=0.042$.

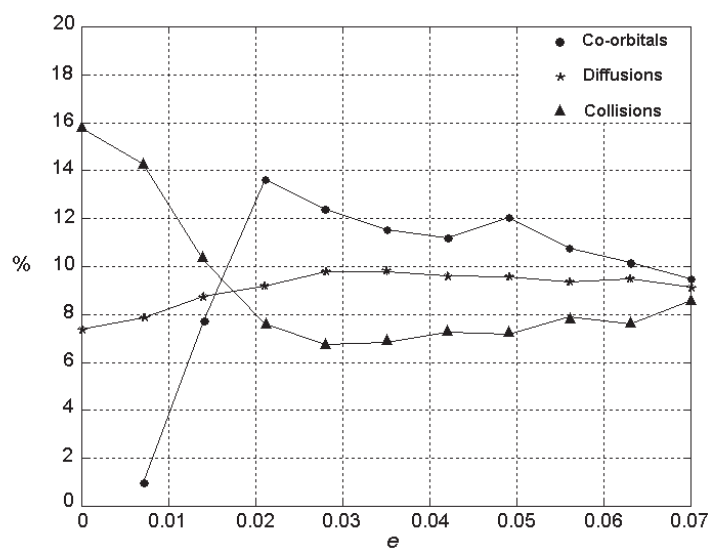

a)

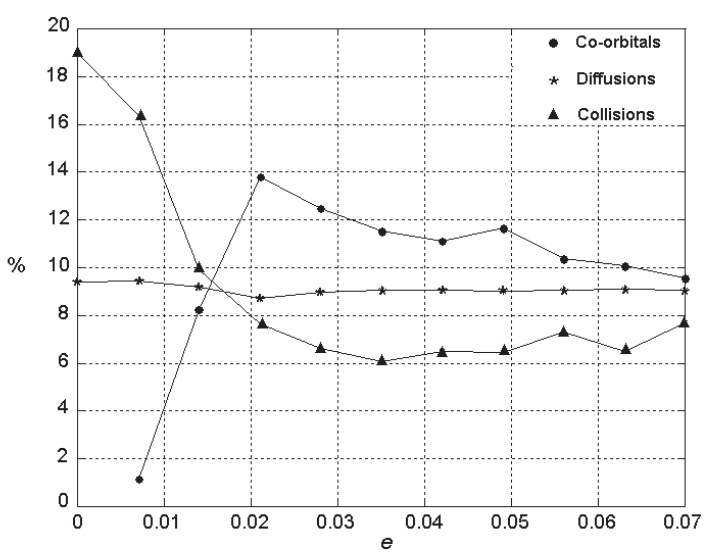

b)

Fig. 6. Distribution of co-orbitals, collisions and diffusions of the whole planetesimal simulation as a function of the secondary body's eccentricity for a mass ratio of $10^{-6}$. The cutoff collision radius is a) $0.05 R_{\mathrm{Hill}}$ and b) $0.005 R_{\mathrm{Hill}}$.

and becomes dominant when its value is above half of the intervals, while there is almost no dependency on the eccentricity of the secondary body (Figs. 6 and 7).

\section{Conclusions}

1. In the present work, we chose the drag model of a primordial solar nebula defined by Murray (1994) and for the dynamic system we used the conditions of Kary \& Lissauer (1995). We studied trajectories of planetesimals whose orbits decay due to gas drag and are perturbed by the gravity of a secondary body on an eccentric orbit with a mass ratio that takes values from $\mu_{2}=10^{-7}$ to $\mu_{2}=10^{-3}$. Each planetesimal ultimately suffers one of three possible fates: (1) trapping in a mean motion resonance with the secondary body; (2) collision with the secondary body increasing the secondary mass; or (3) diffusion, crossing the orbit of the secondary body. We established statistics of the collision rate for two cutoff collision radii $0.005 R_{\text {Hill }}$ and $0.05 R_{\text {Hill }}$ that implies an increase of the mass of the secondary body, and statistics of the diffusion rate that represent the planetesimals crossing the co-orbital region towards the primary body, or being scattered by the secondary body.

2. In the case of captures in the 1:1 resonance with the secondary body, we have shown that, in the process of the capture, an exchange of angular momentum between the planetesimal and the secondary body occurs, the oscillations are reduced and the eccentricity of the planetesimal tends to a certain limit. We also have shown that the eccentricity of the secondary body changes the configuration of the equilibrium points L4 and L5, where the L4 point reappears, even if the drag conditions are over the limit defined by Murray (1994). The configuration with drag and eccentricity increases the attractive power of the region where the L5 point lies beyond its displacement in the direction of the secondary body. Thus there will occur more trappings than in the L4 point and the planetesimals will have smaller dimensions with larger oscillations, increasing the probability of collisions between them.

3. The results show that the mass ratio where the largest number of trappings occurs (8\%) is $\mu_{2}=10^{-6}$ and decreases to less than $1 \%$ for $\mu_{2}=10^{-4}$. When $\mu_{2}=10^{-3}$, trappings in the $1: 1$ resonance do not occur. The eccentricity increases the relative velocity between the secondary body and the planetesimal and decreases the accretion rate per encounter. The optimal value of $k$ for the 1:1 trapping is between 0.9 and 1.25 , representing a meter size planetesimal per AU. The choice of the cutoff collision radius is important for the accretion rate while it seems to be unimportant for the trapping results. The fact that the trappings do not occur when $k$ reaches the limit of 30 means that the size limit of the planetesimal trapping is $0.2 \mathrm{~m}$ per AU. Thus the collision rate is larger $(40 \%$ of 


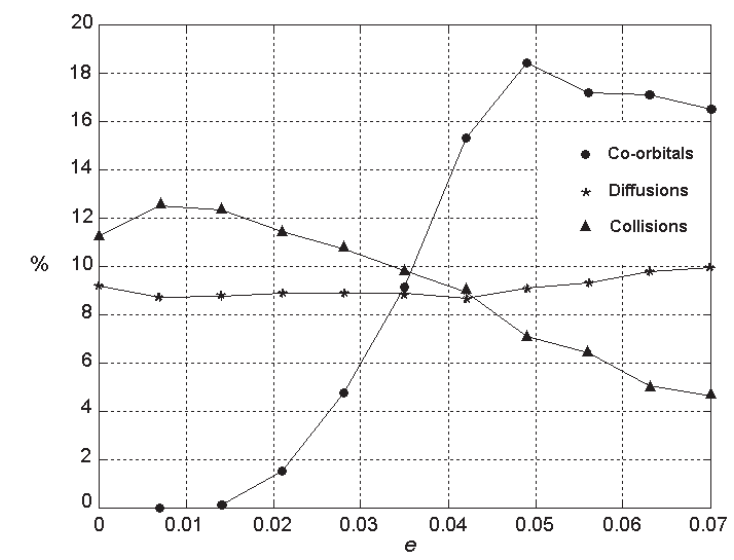

a)

Fig. 7. Distribution of co-orbitals, collisions and diffusions of the whole planetesimal simulation as a function of the secondary body's eccentricity. The cutoff collision radius is $0.05 R_{\mathrm{Hill}}$ and the mass ratio is a) $10^{-5}$ and b) $10^{-4}$.

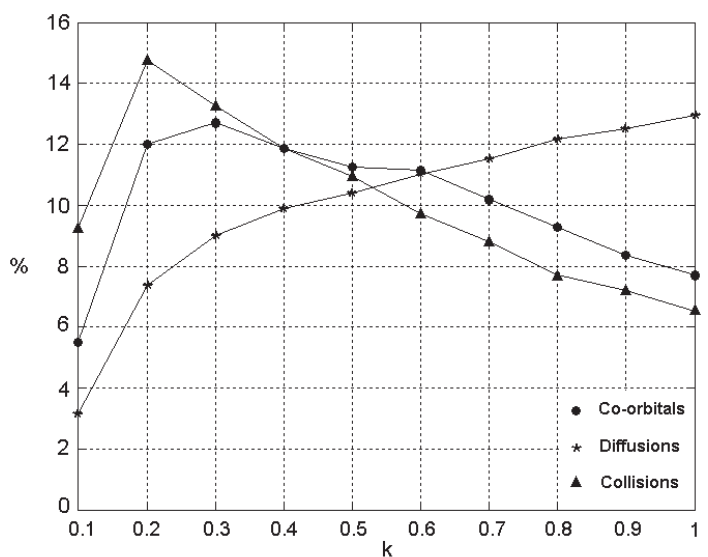

a)

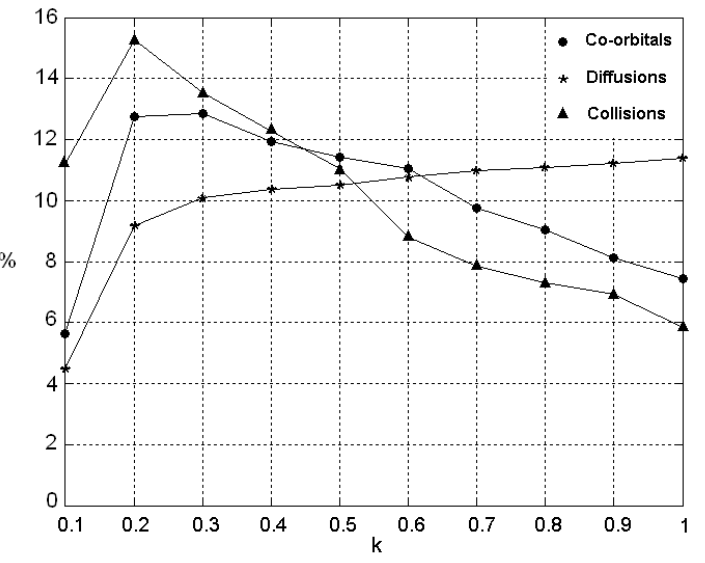

b)

Fig. 8. Distribution of co-orbitals, collisions and diffusions of the whole planetesimal simulation as a function of the drag constant $k$ for a mass ratio of $10^{-6}$. The cutoff radius collision is a) $0.05 R_{\text {Hill }}$ and $\left.\mathbf{b}\right) 0.005 R_{\text {Hill }}$.

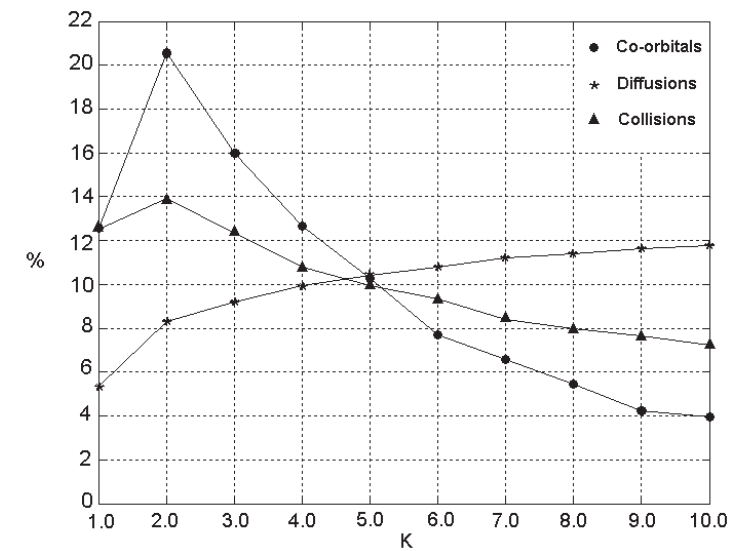

a)

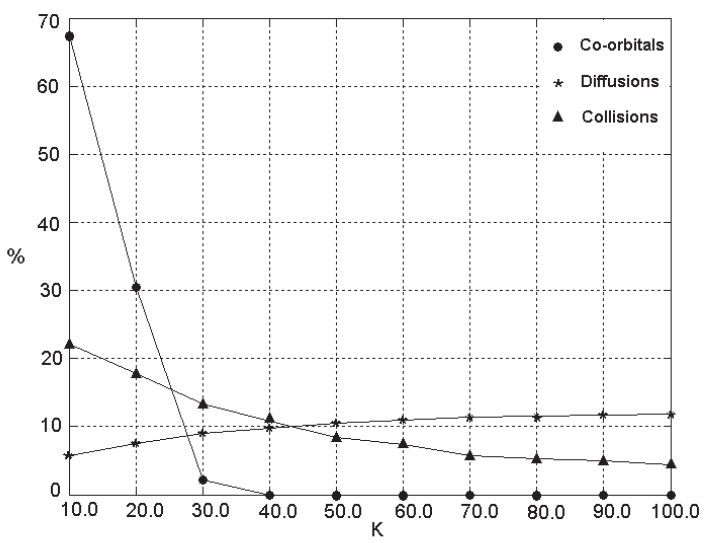

b)

Fig. 9. Distribution of co-orbitals, collisions and diffusions of the whole planetesimal simulation as a function of the drag constant $k$. The cutoff collision radius is $0.05 R_{\text {Hill }}$ and the mass ratio is a) $10^{-5}$ and b) $10^{-4}$.

the cases) when $e=0$ to $e=0.014$. For all the values of the secondary body's eccentricity we have a maximum (15\% of the cases) when $k=0.2$ and a linear decrease from this value when $\mu_{2}=10^{-6}$. This seems to indicate that in the initial formation of the celestial bodies the number of impacts of bodies of large dimension was larger, contributing to a fast mass accretion. We saw that the diffusion rate is directly related to the value of the gas drag constant and is maximum for high values of $k$, showing that gas drag is an efficient process in scattering planetesimals and the trapping of planetesimals in the 1:1 resonance is the less probable fate. These results depends on the specific drag force chosen. Finally, $\mu_{2}=10^{-3}$ is a special case because the cutoff collision radius does not change the results and a particular approach will be 
necessary. Peale (1993) showed that a secondary body larger than 30 terrestrial masses opens a gap in the nebula. This gap decreases the nebula density by up to 10 times when the secondary body reaches 300 terrestrial masses. In a paper in preparation we treat the consequences of this gap for the planetesimal behavior when it decays close to the co-orbital zone.

Acknowledgements. This work was supported by CAPES, CNPq and Fapesp. The authors thank the referee for suggestions that improved the paper.

\section{References}

Brown, E. W., \& Shook, C. A. 1964, Planetary Theory (New York: Dover Publications)

Colombo, G., Lautman, D. A., \& Shapiro, I. I. 1966, J. Geophys. Res., 71, 5705
Kary, D. M., \& Lissauer, J. J. 1995, Icarus, 117, 1

Lagrange, J. L. 1889, Mécanique Analytique (Paris: Gauthier-Villars \& fils), 4th Ed., 2

Malhotra, R. 1993, Icarus, 106, 264

Marzari, F., \& Sholl, H. 1997, Icarus, 131, 41

Morbidelli, A., Levison, H. F., Tsiganis, K., \& Gomes, R. 2005, Nature, 435, 35 Murray, C. D. 1994, Icarus, 112, 465

Namouni, F., \& Murray, C. D. 2000, Celest. Mech., 76, 131

Peale, S. J. 1993, Icarus, 106, 308

Prantl, L. 1952, Essentials of Fluid Dynamics (New York: Hafner Publishing Company), 174

Schuerman, D. 1980, ApJ, 238, 337

Simmons, J. F. L., McDonald, A. J. C., \& Brown, J. C. 1985, Celest. Mech., 35, 145

Yoder, C. F. 1979, Icarus, 40, 341

Weidenschilling, S. J. 1977, MNRAS, 180, 57 